\title{
New Hexagonal CORPS-BFN for Multibeam Antenna Applications
}

\author{
Carlos Biurrun-Quel ${ }^{1,2}$, Antonio Montesano ${ }^{3}$, Iñigo Ederra ${ }^{1,2}$, Juan Carlos Iriarte ${ }^{1,2}$, Carlos del-Río ${ }^{1,2}$ \\ ${ }^{1}$ Antenna Group. Department of Electrical, Electronic and Communication Engineering, Universidad Pública de Navarra, Spain. \\ ${ }^{2}$ Institute of Smart Cities (ISC), Universidad Pública de Navarra, Spain. carlos@unavarra.es \\ ${ }^{3}$ Airbus Defence and Space, Madrid, Spain. antonio.montesano@airbus.com
}

\begin{abstract}
This work presents a new topology of a Coherently Radiating Periodic Structure - Beam Forming Network (CORPS-BFN) and its application for multibeam systems. A unit cell, consisting of a transition from a coaxial input to an intersection of three strip-lines with an angular span of 120 degrees, is proposed and analysed. A periodical replication of the cell gives rise to a uniform layer, allowing a proper matching of the ports of the network. Stacked layers allow in-phase propagation and distribution of the energy through the structure, increasing the number of output ports with each layer.
\end{abstract}

Index Terms-CORPS, Beam Forming Network, Multiple Beam Systems, coaxial to strip-line transition.

\section{INTRODUCTION AND STATE OF THE ART}

In multibeam applications, one of the most frequent limitations is the maximum resolution of the system, given by the dimensions of the radiating elements, which limit the distance between phase-centres of adjacent beams. A useful approach is to substitute electrically large radiating elements with an array of electrically small elements, opening the possibility to overlap beams by feeding different sub-arrays that share these radiating elements. This is especially interesting for applications with reflector systems and cellular coverage [1]. In this paper, a new approach to a CORPS-BFN (Coherently Radiating Periodic Structure Beam Forming Network) [2], [3] is presented. It consists of a hexagonal lattice of alternately up-and-down coaxial connectors at the corners of the hexagons, connected through strip-line waveguides. The proposed structure is able to create an effective overlapping of the beams in the cluster feed, reducing the distance between neighbouring beams, thus enhancing spatial resolution. The radiating elements to be used will depend on specific application requirements. The proposed structure is totally passive and reciprocal, and it could be used in transmission and reception simultaneously. The network could have matching at the input ports better than $30 \mathrm{~dB}$, and the coupling between adjacent input ports is nominally below $14 \mathrm{~dB}$ but the studies carried out in this work suggest that it could be improved up to $30 \mathrm{~dB}$ if required by trading-off resolution. The ideal operation of the network allows the equal distribution of the input power into the three nearest output ports. The number of radiating elements per beam can be increased by stacking several layers of the same network, thus defining a bigger radiating area for each one of the beams, which could be highly overlapped while sufficiently isolated from each other.

The present manuscript is organised as follows: first, an analysis of the proposed structure will be presented. This will be made by increasing gradually the extension of the structure: from a single cell, to a single, infinite layer of periodical cells, to a multi-layer scheme. This analysis will be taken fundamentally from an impedance point of view. A simulation use case with a finite number of cells will be presented. Results will be then discussed and conclusions drawn. Last, some comments regarding their potential applications will be provided and future lines of work will be outlined.

\section{ANALYSIS OF THE PROPOSED STRUCTURE}

\section{A. Unit Cell}

As previously introduced, a new topology of a Coherently Radiating Periodic Structure (CORPS) is proposed in this work. The basic unitary cell of the network is shown in Fig.1. The proposed structure is a 4-port network, whose dimensions could be tuned in order to guarantee an equal power distribution to each branch. The actual dimensions of the different elements (coaxial line, substrate thickness...) lack importance at this point since an analytical study is to be provided. Some relationships among these dimensions, however, will be highlighted, due to their direct relation to the characteristic impedance of transmission lines. The basic structure consists of a transition from a coaxial port to three equal strip-lines placed with an angular span of 120 degrees.

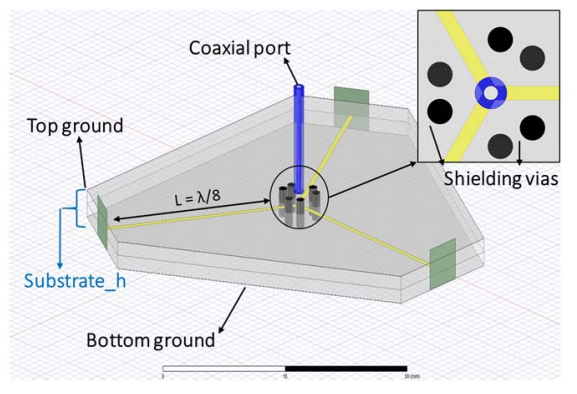

Fig. 1: 3D Representation of the proposed unit cell. Arbitrary dimensions were chosen to illustrate the concept.

(C) 2020 IEEE. Personal use of this material is permitted. Permission from IEEE must be obtained for all other uses, in any current or future media, including reprinting/republishing this material for advertising or promotional purposes, creating new collective works, for resale or redistribution to servers or lists, or reuse of any copyrighted component of this work in other work. 
Some vias surrounding the transition were placed to provide shielding and facilitate the coupling of the fields to the stripline. It is worth noting that, when simulating the standalone cell, it was observed that the cell shows reciprocity (i.e. $\mathrm{S}_{\mathrm{ij}}=\mathrm{S}_{\mathrm{ji}}$ for $\mathrm{i} \neq \mathrm{j}$ ). Let $\mathrm{P} 1$ be the coaxial port and P2...P4 the strip-line ports. Let $Z_{0}$ be the characteristic impedance of the coaxial line and $Z_{s}$ the characteristic impedance of the strip-lines. Since the strip-line ports have been defined as Wave Ports in HFSS, they could be considered as semi-infinite lines, namely loads with impedance $Z_{S}$. The input impedance observed from $\mathrm{P} 1$ is directly obtained by calculating the parallel of the three strip-line impedances. Thus,

$$
Z_{\text {in_coax }}=Z_{S} / 3
$$

If perfect matching $\left(\mathrm{S}_{11}=0\right)$ is desired, relation (2) is obtained straightforwardly.

$$
Z_{S}=3 \cdot Z_{0}
$$

\section{B. Condition of Infinite Periodicity}

Let us assume now an infinite, periodical replication of the cell, connecting each cell to three neighbours. After this periodical transformation, all the ports of the network will be coaxial lines, while the strip-lines will work as interconnections between these coaxial ports. The electrical distance between these ports will be now a quarter of a wavelength. This fact is of great importance, as it will be explained later. The replication of the cell in the aforementioned manner implies the creation of a hexagonal lattice, as depicted in Fig 2.

It is important to note that, within this replication, neighbour coaxial ports will present different orientations on the Z-axis. This means, if the coaxial port in the first cell is pointing upwards, the three nearest neighbours will point downwards. Following this fashion, their neighbours will point upwards, and so on. A first-order in terms of impedance analysis of the network is now proposed.

Given the periodicity condition, since no end of the lattice is considered, it could be asserted that the impedance seen at the input of each strip-line branch is the same, namely $Z_{\text {in. }}$. This impedance will be the result of the mutual influence among every neighbour cell. In other words, it could be stated that each cell will be loaded by the rest of the network. Under the same assumption, the impedance seen at the end of each stripline, namely $\mathrm{Z}_{\mathrm{A}}$ (corresponding to the parallel of the coaxial line and the other two strip lines, connected to the rest of the network) will also be the same at every intersection. For a clearer perspective from the impedance point of view, refer to Fig. 3. Let $Z_{0}$ and $Z_{s}$ be the characteristic impedance of the coaxial line and the strip-line respectively.

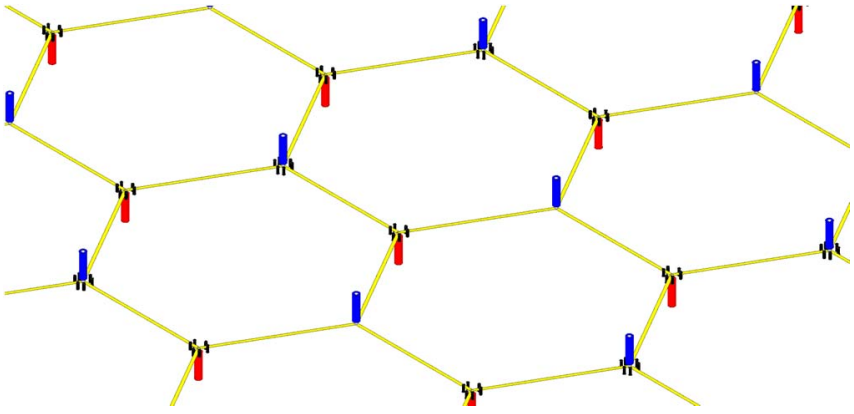

Fig. 2: Overview on the hexagonal lattice formed after the periodical replication of the cell. Blue is used for coaxial ports pointing upwards, while red is used for the ones pointing downwards.

Since the length of the strip-line corresponds to a quarter of a wavelength, the strip-lines work as quarter-wave impedance transformers, following the well-known relationship:

$$
\mathrm{Z}_{\text {in }}=\mathrm{Z}_{\mathrm{S}}^{2} / \mathrm{Z}_{\mathrm{A}}
$$

Where $\mathrm{Z}_{\mathrm{A}}$ is calculated as the parallel impedance between one coaxial port and two strip-lines loaded with the rest of the periodical structure.

$$
\begin{gathered}
Z_{A}=Z_{0} / / Z_{\text {in }} / / Z_{\text {in }} \\
1 / Z_{A}=1 / Z_{0}+1 / Z_{\text {in }}+1 / Z_{\text {in }} \\
Z_{A}=Z_{0} \cdot Z_{\text {in }} /\left(2 \cdot Z_{0}+Z_{\text {in }}\right)
\end{gathered}
$$

Given that, in order to ensure impedance matching at the coaxial ports, $Z_{\text {in }}=3 \cdot Z_{0}$, and substituting (4) in (3), the following relationship between the characteristic impedance of the coaxial lines and the strip-lines is obtained (5).

$$
\mathrm{Z}_{\mathrm{S}}=\mathrm{Z}_{0} \cdot \operatorname{sqrt}(9 / 5)
$$

Furthermore, it can be also checked that:

$$
\mathrm{Z}_{\mathrm{A}}=3 / 5 \cdot \mathrm{Z}_{0}
$$

At this point, since the input impedance seen at each branch is the same, it can be stated that the total power entering the network from an only coaxial input port is divided equally among the three strip-lines. Following this assumption, the power delivered to the neighbour coaxial ports can be calculated as the power delivered to a $Z_{0} \Omega$ load in a current divisor with three parallel impedances: $Z_{0} / / Z_{\text {in }} / /$ $\mathrm{Z}_{\text {in }}$ from the point of view of $\mathrm{Z}_{\mathrm{A}}$. 


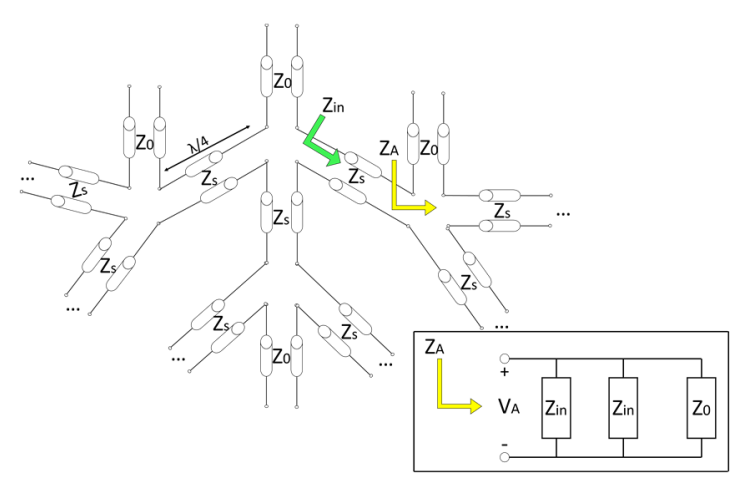

Fig. 3: Sketch of periodicity condition and impedance point of view. Inset contains s scheme of the current division present at every intersection, from the point of view of a strip-line

Refer back to Fig. 2 and the inset in Fig. 3 for a clearer perspective. Here, an auxiliary voltage $\mathrm{V}_{\mathrm{A}}$ could be defined from the relation between power and voltage (7a). Subsequently, it can be obtained that the power delivered to the load $Z_{0}$ corresponds to $3 / 5$ of the power available at the input strip-line. Therefore, it is concluded that the power delivered to each coaxial port, neighbour to the input coaxial, will be:

$$
\begin{gathered}
\mathrm{P}=\mathrm{V} \cdot \mathrm{I}=\mathrm{V}^{2} / \mathrm{Z} \\
\mathrm{P}_{\mathrm{Z} 0}=\mathrm{V}_{\mathrm{A}}^{2} / \mathrm{Z}_{0}=\mathrm{P}_{\mathrm{A}} \cdot \mathrm{Z}_{\mathrm{A}} / \mathrm{Z}_{0}=1 / 3 \mathrm{P}_{\mathrm{T}} \cdot 3 / 5=1 / 5 \mathrm{P}_{\mathrm{T}}
\end{gathered}
$$

Where $\mathrm{P}_{\mathrm{T}}$ stands for the total input power coming from the first coaxial port. Until now, the amount of power delivered to each branch from the coaxial port ( $1 / 3$ each) and the amount of power delivered to each neighbour port (three-fifths of the available power at each branch, namely one-fifth of the total power) have been determined. From these results, it can be deduced that $6 / 15$ of the total power (one per strip-line branch after the 3 first coaxial ports) are being delivered to the rest of the network. In the next intersection, as stated in (7b), 3/5 of the power is delivered to the coaxial port $(1 / 25$ of the total power, namely $-14 \mathrm{~dB}$ ). This port could be another input to the system and this value will be the nominal isolation between in-plane consecutive coaxial ports (this means, consecutive coaxial ports with the same orientation in the $\mathrm{Z}$-axis). It is important to remark that this first-order analysis does not include multipath contributions across the network, which will contribute either constructively or destructively, although they might be significantly small. Another fact that should be borne in mind is that an actual implementation will imply a finite extension of the hexagonal lattice, which will impose an impedance mismatch on the outermost coaxial ports. In those cells, at least one of the strip-line branches is kept open, being the source of the impedance mismatch. This would be solved by placing a load impedance $Z_{\mathrm{A}}$, in straight relation to $Z_{0}$, as defined by (6) after the quarter wave transformer. Nevertheless, as it will be discussed in next section, simulation has proved that, if the network is sufficiently large, the effect on the impedance matching for the inner ports will be imperceptible.

\section{PRoposal of Use CASE}

In order to provide an example of the performance of the network, a single, finite layer of the structure, as depicted in Fig. 4, was simulated in ADS (Advanced Design System). The coaxial ports were modelled by lumped ports with $Z_{0}=50 \Omega$. The L- Band was chosen as the band of operation and a reference frequency $\mathrm{f}_{0}$ of $1.5 \mathrm{GHz}$ was chosen to design the quarter wave transformers. The fact that the topology is composed of resonant elements infers the resonant behaviour of the network. Since simulating an infinitely periodical structure was unfeasible, a reasonably large structure with 121 ports was simulated. Port 1 will be the first input port considered. By applying (5), a strip-line width of $1.19 \mathrm{~mm}$ was obtained with ADS' LineCalc tool. A further optimization, however, prompted a value of $1.08 \mathrm{~mm}$ providing the most resonant behaviour in terms of S11. Nevertheless, it was observed for both cases that the transmission to next ports (for example, from Port 1 to Ports 2, 6 and 10 in Fig. 4) was around $-6.5 \mathrm{~dB}$, slightly higher than the theoretical result presented in the previous section, presumably due to the influence of the multipath contributions. As a result, a lower amount of power is delivered to the rest of the network, increasing the isolation between consecutive ports to roughly $-16 \mathrm{~dB}$.

Another interesting finding is the isolation between nonconsecutive ports. Here, we can distinguish between two types of non-consecutive ports: the ones located at the three symmetry axis (ports 15, 19, 23, 27, 31 and 35), and the ones which are not $(17,21,25,29,33$ and 37) - See Fig. 7 for a clearer view. It has been seen that the isolation between port 1 and ports of the second type presents higher values (up to $35 \mathrm{~dB}$ ) than the rest (around $20 \mathrm{~dB}$ ). Depending on the isolation required between input ports, a different configuration shall be chosen, presenting a trade-off between isolation and the distance among input ports (i.e. distance between the centres of the different overlapped beams). All these parameters are represented in Fig. 5. This study does not yet involve an actual application with stringent requirements in terms of bandwidth, but it can be observed that several fractional bandwidths could be defined, as for example a BW$1 \mathrm{~dB}$ of $66 \%$ in terms of transmission to consecutive ports and reflection coefficient under $10 \mathrm{~dB}$.

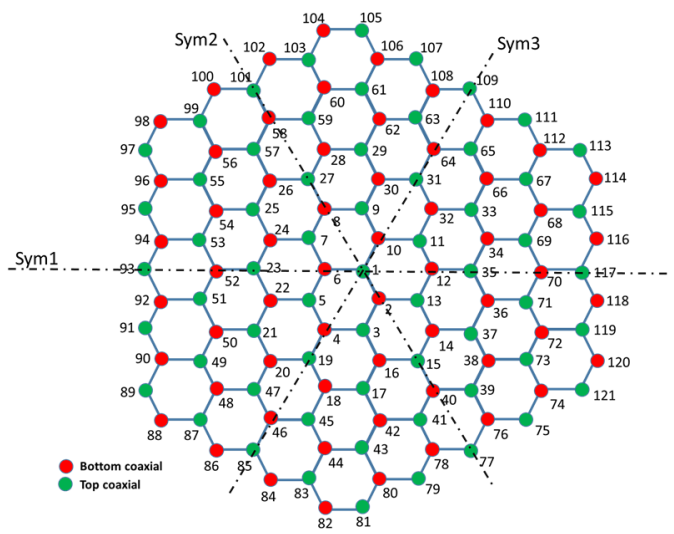

Fig. 4: Diagram of the simulated structure in ADS, with 121 coaxial inputs. 


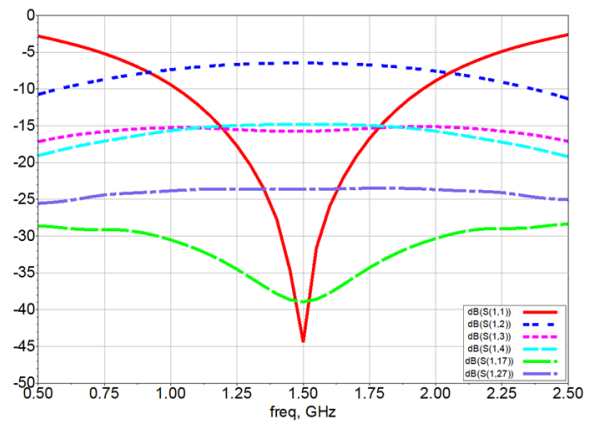

Fig. 5: Input port's reflexion, transmission and isolation between input and output ports calculated by ADS

\section{Stacked N-LAYER AND Multi-BeAm Operation}

Up to this point, the main features and behaviour of the network have been presented and its properties under a periodicity condition have been discussed. The next step involves the stacking of several layers of the hexagonal lattice, as sketched in Fig. 6. With this regard, it is important to note that in Chapter II it was stated that the coaxial ports are arranged with alternately directions. This feature will allow the interconnection of the layers and both the vertical longitudinal propagation of the energy across the structure. Since all layers are equal, it can be assumed that the energy propagation from one port to its neighbours will follow the same fashion. Thus, the higher the number of layers, the more spread the power will be. Refer now to Fig. 7, at the bottom of this document, to see an example representation of the power distribution of an $\mathrm{N}=4$ scenario for a simplified case where only power travelling upwards is considered and power spread farther than 2 ports away from the input ports at each layer is neglected. As seen in Chapter III, the theoretical power division of one third at each neighbour is not achieved. For this reason, and in order to simplify the scheme in Fig. 7, a "k" factor is employed to represent the proportion of power delivered to each neighbour. Here, blue circles represent the inputs at each layer, while yellow circles represent the output ports at each layer. Green circles represent coaxial lines pointing downwards and red circles correspond to the ones pointing upwards at $\mathrm{N}=1$ (and every odd layer number). For $\mathrm{N}=2$ and every even layer number their orientation shall be exchanged in order to follow an upward propagation (an output in layer $\mathrm{N}-1$ is an input in layer $\mathrm{N}$ ). The power is distributed within each layer, concentrating its most part at the central position with respect to the input port at Layer 1. It can be seen that this network allows feeding a triangular/hexagonal distribution of radiating elements separated less than a half of a wavelength. Regarding multibeam operation, let us refer the Fig. 8, where the outputs of a 4-layer network are shown. Here, seven different beams have been represented by circles of different colours, each beam being fed at layer 1 from different coaxial ports. Each circle circumscribes up to 19 radiating elements that individually present the amplitude distribution seen in Fig.7. It is assumed that they are in phase. The central, green circle represents the elements conforming the beam generated by port 1 .

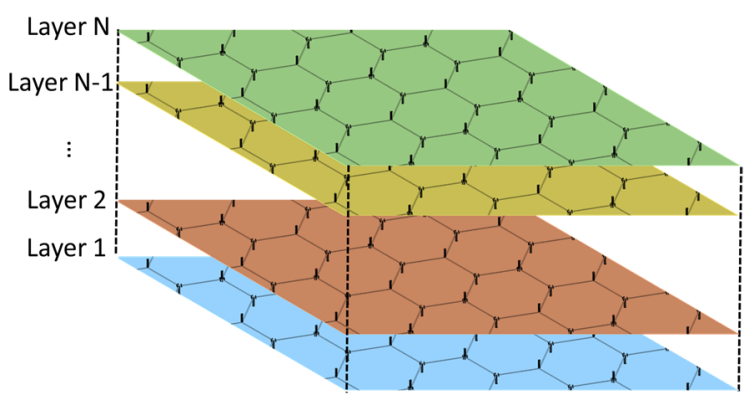

Fig. 5: Sketch of stacking layers. Coaxial ports pointing upwards in N-1 layer correspond to coaxial ports pointing downwards in layer $\mathrm{N}$.

The other six circles correspond to the radiating elements illuminated when feeding the network from ports $15,19,23$, 27, 31 and 35. An effective overlapping of 10/19 elements would be achieved in this sample case.

\section{CONCLUSIONS, APPLICATIONS AND FUTURE WORK}

A new topology for a CORPS-BFN based on a multi-layer, hexagonal lattice with coaxial input and output ports has been presented. The canonical features of the fundamental topology have been discussed, showing an interesting, remarkable potential in multibeam applications to allow overlapping different beams in the focal plane of a reflector system while keeping the isolation between input ports under a reasonable level. The use case presented in this work has shown the possibility to trade off isolation between input ports and the resolution of the system.

Future lines of work will involve the further study of the proposed topology. Deeper study of the multipath contributions and the differences between simulation and theoretical results shall be made. The trade-off between perfectly matched inputs, power delivered to the coaxial ports and to the rest of the network, will be analysed in order to develop a more robust model, attempting to maximize the power transmission to the neighbour ports while ensuring a reasonable isolation. In addition, a broader study of the phase in the network shall be made, since the geometry of the network itself constrains the phase being fed at each radiating element.

\section{ACKNOWLEDGMENT}

This work was supported by the Spanish Ministry of Science, Innovation and Universities (FPU18/00013).

\section{REFERENCES}

[1] D. Betancourt, C. Del-Rio-Bocio, "Multi-beam applications of CORPS BFN: Reflector antenna feeding system", 30th ESA Antenna Workshop European Space Agency (ESA) - European Space Research and Technology Centre (ESTEC), 2008.

[2] R. Garcia, D. Betancourt, A. Ibanez, and C. del-Rio, "Coherently radiating periodic structures (CORPS): a step towards high resolution imaging systems?," in 2005 IEEE Antennas and Propagation Society International Symposium, vol. 4B, pp. 347-350.

[3] D. Betancourt and C. del Rio Bocio, "A Novel Methodology to Feed Phased Array Antennas,” IEEE Trans. Antennas Propag., vol. 55, no. 9, pp. 2489-2494, Sep. 2007. 


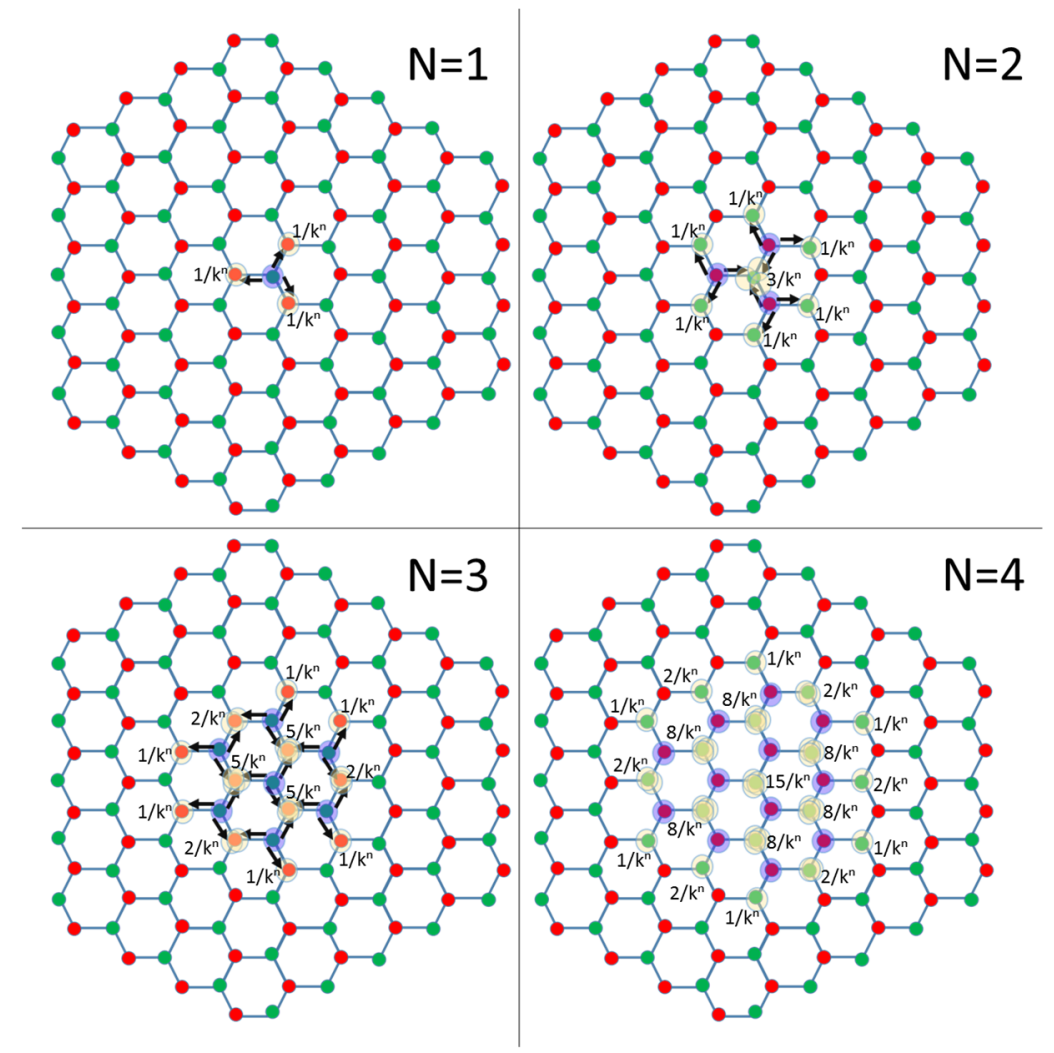

Fig. 6: Example of power distribution on a 4-layer CORPS with a periodic layer for the ideal case. Light blue circles represent the sources on each layer. Light yellow circles represent the receiving nodes (output ports) on each layer.

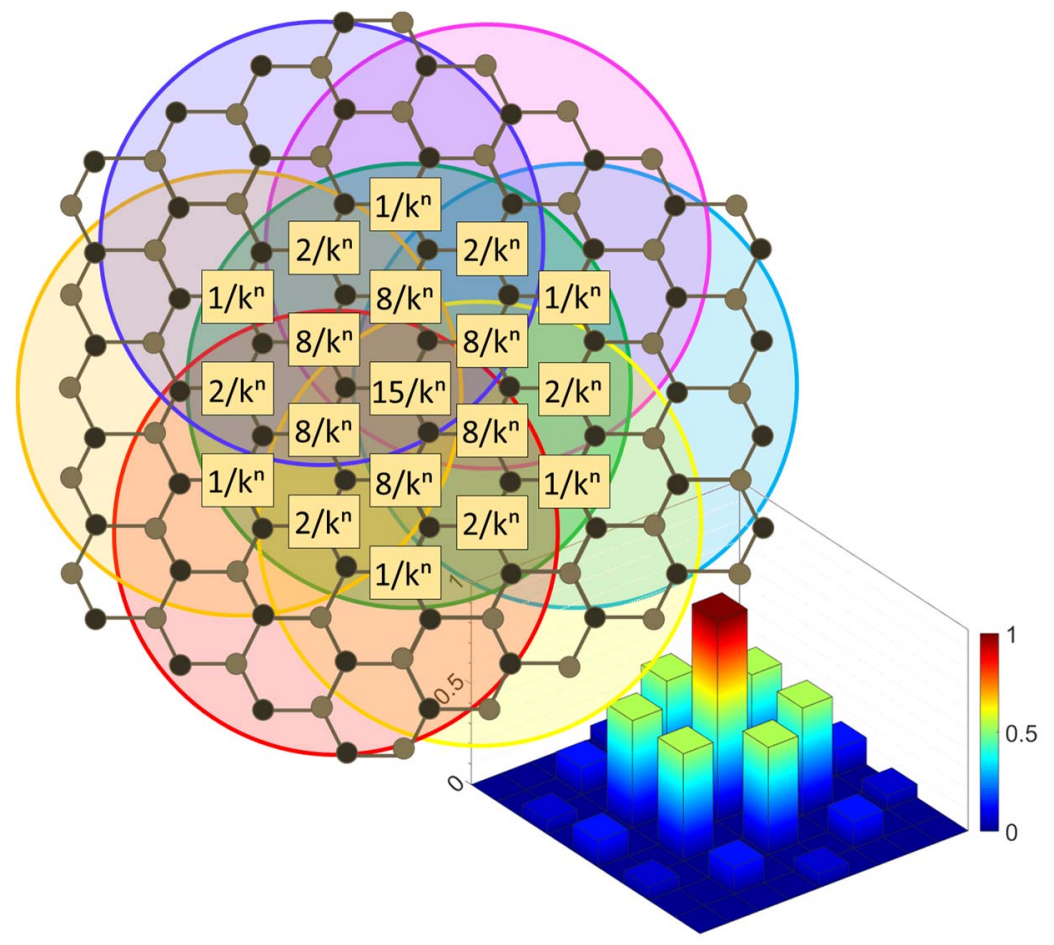

Fig. 8: In this scenario, a 4-layer network could be used to feed an hexagonal/triangular array of 19 radiating elements per beam, where two adyacent beams share 10 elements 